\title{
ARTICLE
}

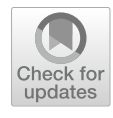

Cite as

Nano-Micro Lett.

(2021) 13:96

Received: 18 November 2020

Accepted: 23 January 2021

Published online: 22 March 2021

(C) The Author(s) 2021

\section{Targeted Sub-Attomole Cancer Biomarker Detection Based on Phase Singularity 2D Nanomaterial-Enhanced Plasmonic Biosensor}

\author{
Yuye Wang ${ }^{1,2}$, Shuwen Zeng ${ }^{2,3} \bowtie$, Aurelian Crunteanu ${ }^{2}$, Zhenming Xie ${ }^{1}$, \\ Georges Humbert ${ }^{2}$, Libo $\mathrm{Ma}^{4}$, Yuanyuan $\mathrm{Wei}^{1}$, Aude Brunel ${ }^{5}$, Barbara Bessette ${ }^{5}$, \\ Jean-Christophe Orlianges ${ }^{2}$, Fabrice Lalloué ${ }^{5}$, Oliver G. Schmidt ${ }^{4}$, Nanfang $\mathrm{Yu}^{3}$, \\ Ho-Pui Ho ${ }^{1 凶}$
}

\section{HIGHLIGHTS}

- A zero-reflection-induced phase singularity is achieved through precisely controlling the resonance characteristics using two-dimensional nanomaterials.

- An atomically thin nano-layer having a high absorption coefficient is exploited to enhance the zero-reflection dip, which has led to the subsequent phase singularity and thus a giant lateral position shift.

- We have improved the detection limit of low molecular weight molecules by more than three orders of magnitude compared to current state-of-art nanomaterial-enhanced plasmonic sensors.

ABSTRACT Detection of small cancer biomarkers with low molecular weight and a low concentration range has always been challenging yet urgent in many clinical applications such as diagnosing early-stage cancer, monitoring treatment and detecting relapse. Here, a highly enhanced plasmonic biosensor that can overcome this challenge is developed using atomically thin two-dimensional phase change nanomaterial. By precisely engineering the configuration with atomically thin materials, the phase singularity has been successfully achieved with a significantly enhanced lateral position shift effect. Based on our knowledge, it is the first experimental demonstration of a lateral position signal change $>340 \mu \mathrm{m}$ at a sensing interface from all optical techniques. With this enhanced plasmonic effect, the detection limit has been experimentally demonstrated to be $10^{-15}$ mol $\mathrm{L}^{-1}$ for TNF- $\alpha$ cancer marker, which has been found in various human diseases including inflammatory diseases and different kinds of cancer. The as-reported novel integration of atomically thin $\mathrm{Ge}_{2} \mathrm{Sb}_{2} \mathrm{Te}_{5}$ with plasmonic substrate, which

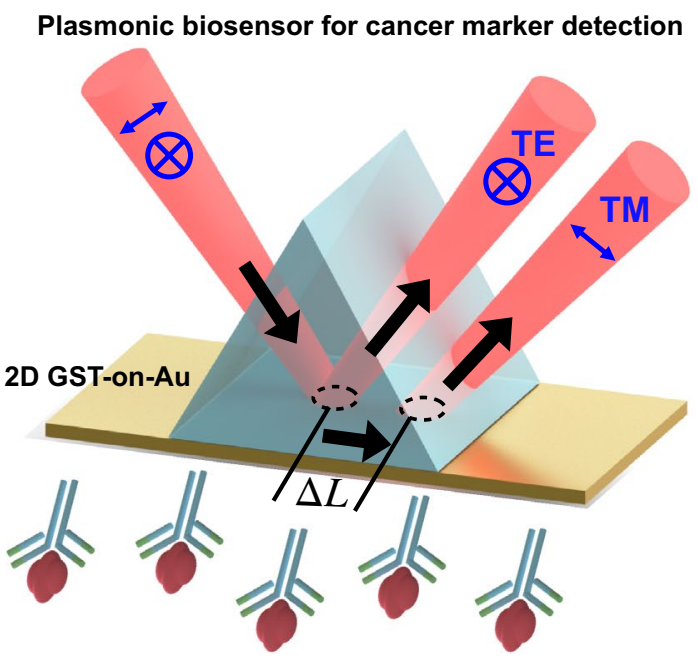
results in a phase singularity and thus a giant lateral position shift, enables the detection of cancer markers with low molecular weight at femtomolar level. These results will definitely hold promising potential in biomedical application and clinical diagnostics.

KEYWORDS 2D nanomaterials; Cancer marker detection; Phase singularity; Surface plasmon

\footnotetext{
Shuwen Zeng, zeng@xlim.fr; Ho-Pui Ho, aaron.ho@cuhk.edu.hk

Department of Biomedical Engineering, The Chinese University of Hong Kong, Shatin, New Territories, Hong Kong, People's Republic of China

2 CNRS, XLIM Research Institute, UMR 7252, University of Limoges, 123, Avenue Albert Thomas, Limoges, France

3 Department of Applied Physics and Applied Mathematics, Columbia University, New York City, NY, USA

4 Institute for Integrative Nanosciences, IFW Dresden, Helmholtzstr. 20, Dresden, Germany

5 Faculty of Medicine, University of Limoges, EA3842-CAPTuR, GEIST, 2 rue du Dr Marcland, Limoges, France
} 


\section{Introduction}

To promote early-stage diagnostics of a variety of human diseases, the detection of specific biomarkers at extremely low concentration levels has attracted a lot of attention over these years [1-3]. Among the various biomarkers, tumor necrosis factor TNF- $\alpha$ has been studied intensively and proved to play a central role in mammalian immunity and cellular homeostasis $[4,5]$. TNF- $\alpha$ is a key mediator in pro-inflammatory responses. It is also involved in various cell activities including cellular communication, cell differentiation and cell death [6]. These regulatory functions have made TNF- $\alpha$ an important biomarker for monitoring a variety of human diseases, including inflammatory disorder such as bowel disease [7], osteoarthritis [8, 9], rheumatoid arthritis [10] as well as malignant tumors such as oral [11] and breast cancer $[12,13]$, etc. The detection of biomarkers, particularly TNF- $\alpha$, is certainly of great significance to healthcare efficacy through early diagnosis and monitoring of life-threatening diseases. However, the concentration level of this biomarker is extremely low, typically $\sim 20 \mathrm{pg} / \mathrm{mL}$ in a healthy human $[14,15]$, thus making detection of this molecule a significant challenge. Moreover, the molecular weight of TNF- $\alpha(\sim 17 \mathrm{kDa})$ is one order of magnitude lower than many common biomarkers such as argonaute proteins [16] ( $100 \mathrm{kDa})$ and carcinoembryonic antigen [17] ( $\sim 180 \mathrm{kDa})$, which further increases the level of difficulty.

Conventional detection techniques include gel electrophoresis, enzyme-linked immunosorbent assay (ELISA) and fluorescence-based detection [18, 19], etc. However, traditional detection methods are usually time-consuming and require complex operations with adequate transducing elements such as fluorescent dyes or expensive enzymes [20]. Over these years, some cutting-edge technologies including electrochemical approaches [21] and microfluidic-based approaches [22] have been developed to enable more effective and sensitive cancer marker detection. More recently, several research groups have exploited mass spectrometry (MS) based on optimized nanoparticles as matrix materials to enable fast detection with high selectivity and sensitivity [23-25]. This MS-based detection can directly detect small metabolites in human serum without any purification in advance [26, 27]. However, bulk instrumentation is needed in this case, which largely limits the application in on-site detection. As a result, there is still much room for improvement in terms of low-cost, convenient and ultrasensitive biodetection as well as point-of-care diagnostics.

Due to its label-free, real-time and low-cost detection merits, the surface plasmon sensing technique has been exploited for a range of biosensing applications [28-30]. However, for target analytes that have low molecular weights (less than 400 Dalton), plasmonic sensors still face the challenges to compete in terms of detection sensitivity [31]. Compared to traditional plasmonic biosensor designs based on angle [32,33] or wavelength [34-36] interrogation, the phase detecting approach has been shown to improve the sensitivity limit by a few orders of magnitude [37, 38]. Unlike the moderate change in intensity or wavelength, the optical phase experiences an abrupt change when the reflection reaches almost zero. Furthermore, the phase detection can also provide lower noise and allows for versatile signal-processing possibilities [39]. Recently, with increasing efforts to explore the concept of "point of darkness," which represents the zero-reflection point, several research groups have exploited plasmonic metamaterials that exhibit topologically protected darkness for the design of biosensing devices offering radically enhanced sensitivity due to extremely steep phase variations [37, 40, 41]. Further, a higher order of the phase signal, the lateral position shift, may bring further improvement in sensitivity. Since zeroreflection can lead to a singular behavior of the phase in Fourier space, the resulted sharp phase jump will then induce a giant lateral position shift, making it an excellent choice for sensing biomolecules at very low concentrations [42, 43].

In this work, we have significantly enhanced the performance of a SPR biosensing platform by adding an atomically thin phase change material to induce a giant lateral position shift called Goos-Hänchen (GH) shift, which in turn leads to the detection of TNF- $\alpha$ cancer biomarkers at sub-attomole level. We tuned the atomically thin $\mathrm{Ge}_{2} \mathrm{Sb}_{2} \mathrm{Te}_{5}$ (GST) nanomaterials, which have a high absorption rate [44] in visible and near-infrared wavelengths, to achieve zero-reflection at plasmon resonance. The zero-reflection phenomenon can result in a strong phase singularity. This zero-reflectioninduced phase singularity is known to be challenging to achieve in previous plasmonic nanostructures $[37,45]$. The higher-order mode of the phase signal, i.e., the lateral position shift, was found to be much larger than other signal modalities reported in recent years [46-49]. The plasmonic sensing device reported herein exhibits a detection limit of $10^{-15} \mathrm{~mol} \mathrm{~L}^{-1}(1 \mathrm{fM})$ for TNF- $\alpha$ biomarkers and $10^{-14} \mathrm{~mol}$ 
$\mathrm{L}^{-1}(10 \mathrm{fM})$ for small biotin molecules $(\mathrm{MW}=244.31 \mathrm{Da})$. This sub-attomole detection level is a significant improvement compared to other SPR designs [50, 51]. The maximum experimental lateral position shift triggered in our device is $341.90 \mu \mathrm{m}$, which to our best knowledge is the largest value ever reported. For detecting small biomolecules and cancer markers at femtomolar concentration levels, the sensing signal was around $10 \mu \mathrm{m}$, which is quite readily measured by an interrogation setup. In summary, the proposed scheme has been shown to be capable of sensing extremely small refractive index (RI) changes, which is of great interest for label-free biosensing sensing application and clinical diagnostics.

\section{Experimental Methods}

\subsection{Methodology of the Enhanced Plasmonic Sensor}

We have significantly enhanced the sensitivity of a plasmonic sensing system by engineering an atomically thin GST layer to the plasmonic substrate. The designed atomically thin GST-on-Au plasmonic biosensing scheme was based on the Kretschmann configuration as shown in Fig. 1a. For the sensing layer design, an atomically thin layer of 2D GST material is uniformly deposited on the top of the $\mathrm{Au}$ thin film. The GST phase change materials are known to have a higher absorption rate in visible and near-infrared regions than some other 2D materials such as graphene, $\mathrm{MoS}_{2}$ and $\mathrm{WS}_{2}$ [52-54]. As a result, with careful optimization of the thickness of GST layer, a condition very close to zero-reflection can be achieved. Under this near zeroreflection phenomenon, we can observe not only a fast drop in reflected light intensity, but also an extremely sharp phase change at the resonance angle, which can be exploited to greatly enhance the sensitivity based on plasmon resonances. The lateral position shift is a higher-order optical signal of the phase singularity (See Eq. (3)) and is readily detectable by our plasmonic sensing setup. It is worth noting that only $p$-polarized light will have this giant phase singularityinduced lateral position shift under the plasmonic excitation while s-polarized light remains unaffected (Fig. 1b). Therefore, $s$-polarized light serves as a reference here to eliminate environmental disturbances and can be used to significantly improve the signal-to-noise ratio of the measurement. The differential signal acquired by the position sensor between the $p$ and $s$ polarizations can provide high signal-to-noise measurements. The largest lateral position shift is achieved when the sharpest phase change occurs, which corresponds to the minimum reflectance point within the surface plasmon resonance dip. Our theoretical results have shown that the lateral position shift is inversely correlated with the reflectivity (Fig. S1). In order to achieve the largest lateral position shift, we have enhanced the absorption characteristics to near zero-reflectance $\left(\sim 10^{-6}\right)$ by adding an atomically thin 2D phase change material. Based on the theoretical model, the optimized thicknesses of the $2 \mathrm{~nm}$ GST layer can lead to minimum reflectance, i.e., maximum sensitivity. The lateral position shift of both Au-only substrate and our atomically thin GST-on-Au substrate was simulated. It is clearly shown in Fig. 1c that the addition of atomically thin GST material can lead to an extremely giant maximum lateral position shift up to $2107.33 \mu \mathrm{m}$ at the resonance angle, which is nearly 100 times larger than that associated with the case of using Au-only substrate. Here, we also demonstrated the extreme singularities in phase of the reflected light enhanced by GST-on-Au substrate in Fig. 1d. Compared to Au-only substrate, the phase change tends to be much sharper, which indicates the radically enhanced sensitivity based on our GST-on-Au substrate. Moreover, the electric field distribution on this 2D GST-on-Au sensing substrate at the resonance angle was also studied using finite element analysis (FEA) (COMSOL Multiphysics 5.2) (Fig. S2a). The large electric field enhancement at the sensing interface also demonstrated the enhanced plasmonic resonance. These results have shown that the atomically thin 2D GST layer will offer a superior sensitivity enhancement.

\subsection{Optimization of the 2D GST Thickness on the Plasmonic Sensing Substrate}

In a Kretschmann configuration, the reflection coefficient of two adjacent layers takes the form of

$r_{i, i+1}=\frac{Z_{i}-Z_{i+1}}{Z_{i}+Z_{i+1}}$

where $Z_{i p}=\frac{\varepsilon_{i}}{k_{i}}$ for $p$ polarization and $Z_{i s}=k_{i}$ for $s$ polarization. $\varepsilon_{i}$ represents the complex dielectric constants of the $\mathrm{i}$-th layer and $k_{i}=k_{0} \sqrt{\varepsilon_{i}-\varepsilon_{1} \sin ^{2} \theta_{c}}$, in which $k_{0}$ is the wave vector of the optical wave in free space and $\theta_{c}$ is the incident angle.

For a sensing substrate with $m$ layers, we have 


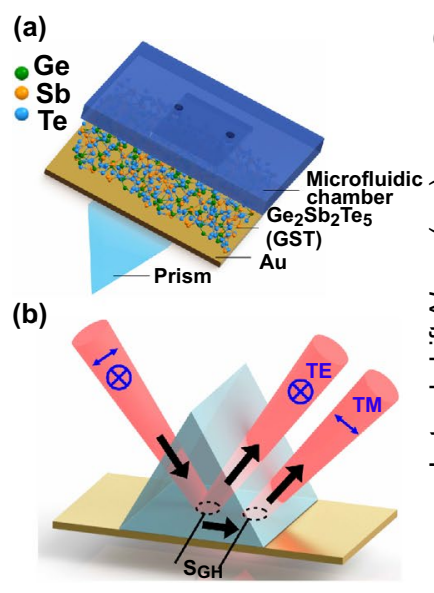

(c) 2D GST-on-Au substrate
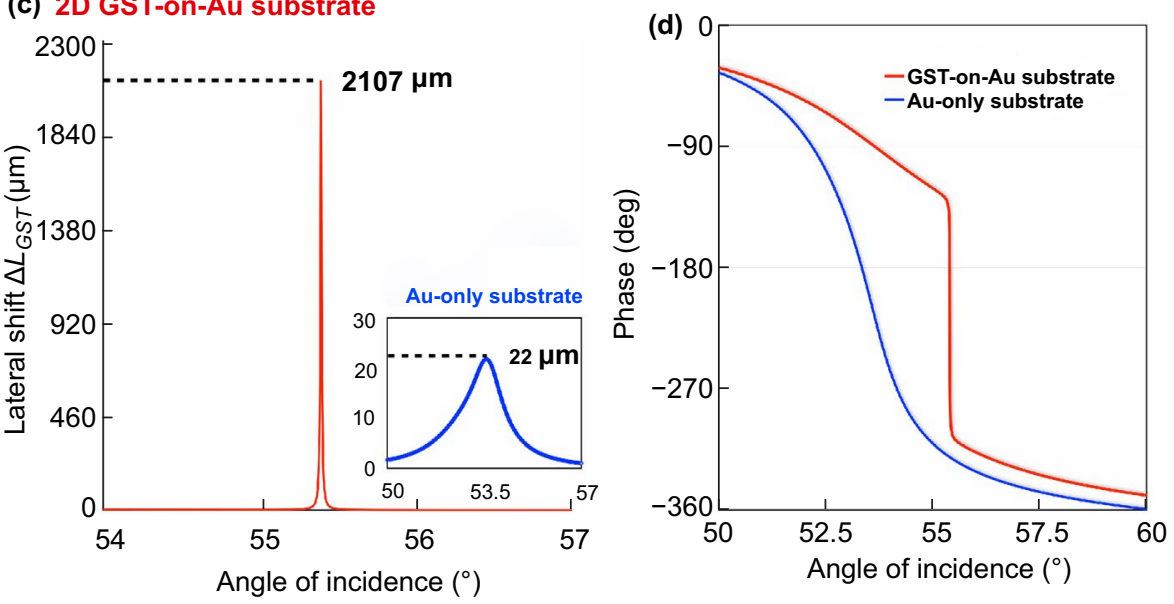

Fig. 1 a Schematic of the sensing substrate based on GST-gold metastructures. b Schematic diagram of the giant lateral position shift induced by the GST-on-Au substrate. Simulation results of $\mathbf{c}$ lateral position shift and d optical phase signal change based on 2D GST-on-Au substrate and Au-only substrate

$r_{m-2, m}=\frac{r_{m-2, m-1}+r_{m-1, m} \exp \left(2 i k_{m-1} d_{m-1}\right)}{1+r_{m-2, m-1} r_{m-1, m} \exp \left(2 i k_{m-1} d_{m-1}\right)}$

Then we subsequently calculated $r_{m-3, m}, r_{m-4, m} \ldots$ until we get $r_{1, m}$, which is the reflectivity of the substrate based on this metastructure.

From Fresnel's equations, the complex reflection coefficients can be expressed as $r_{p(s)}=\left|r_{p(s)}\right| \exp \left(i \phi_{p(s)}\right)$ for $p$ and $s$ polarizations in which $\phi_{p(s)}$ represents the phase of both polarizations. According to the stationary phase approach [55], the lateral position shift represents the higher order mode of the phase signal $[41,56,57]$ and can be determined through the following equation [58]:

$\Delta L=-\frac{1}{k_{0}} \frac{\partial \phi}{\partial \theta}$

If we determine the reflection coefficients through the standard characteristic matrix approach [59,60], the lateral position shift can also be expressed as:

$\Delta L=-\frac{\lambda}{2 \pi\left|r_{p(s)}\right|^{2}}\left(\operatorname{Re}\left(r_{p(s)}\right) \frac{d \operatorname{Im}\left(r_{p(s)}\right)}{d \theta}-\operatorname{Im}\left(r_{p(s)}\right) \frac{d \operatorname{Re}\left(r_{p(s)}\right)}{d \theta}\right)$

where $R e$ is the real part and $I m$ is the imaginary part. Based on the above equations, the optimized thickness of 2D GST layer for achieving the minimum reflectance and maximum lateral position shift can therefore be calculated.

\subsection{Materials and Methods}

\subsubsection{Chemicals}

Glycerol, absolute ethanol, lyophilized Bovine Serum Albumin (BSA) powder, lyophilized biotin powder, and (3-Aminopropyl) trimethoxysilane (APTMS), Tumor necrosis factor-alpha (TNF- $\alpha$ ) antigen powder, Monoclonal AntiTNF antibody were purchased from Sigma Aldrich, France. Colloidal solutions with uniformly dispersed single $\mathrm{MoS}_{2}$ nanosheets were purchased from Ossila Ltd, UK.

\subsubsection{Device Fabrication}

$\mathrm{Ge}_{2} \mathrm{Sb}_{2} \mathrm{Te}_{5}$ (GST) and Au layers of the sensing substrate were fabricated by MP300 DC magnetron sputtering equipment (Plassys-Bestek, France) using stochiometric 2" diameter GST and Au (high purity targets $99.99 \%$, Neyco Vacuum \& Materials, France) targets on glass substrates, respectively. The deposition chamber was pumped down to $2 \times 10^{-6} \mathrm{mb}$ prior to the deposition. The deposition took place under Ar atmosphere (60 sccm flow rate) at $5 \times 10^{-3}$ $\mathrm{mb}$ and $1 \times 10^{-2} \mathrm{mb}$ partial pressures, respectively, using DC magnetron powers between 25 and $55 \mathrm{~W}$. 


\subsubsection{Biosample Preparation}

Glycerol solutions with concentrations from 1-5\% (weight ratio) were prepared. All the solvents used here are deionized water. BSA and biotin solutions with concentrations from $10 \mathrm{fM}$ to $10 \mu \mathrm{M}$ were prepared by serial dilution (1:100). Antibody solutions with $10 \mathrm{pM}$ concentration were diluted several times from originally $0.5 \mathrm{mg} \mathrm{mL}^{-1}$ Monoclonal Anti-TNF solutions. TNF- $\alpha$ human lyophilised powder was reconstituted to a concentration of $0.5 \mathrm{mg} \mathrm{mL}^{-1}$ and further diluted to cancer marker solutions with concentrations from $1 \mathrm{fM}$ to $1 \mathrm{nM}$.

\subsubsection{Surface Functionalization}

The 2D GST-on-Au substrate was first dipped in ethanol and deionized water, followed by drying under nitrogen for clean usage. The GST-on-Au substrate was first immersed in $1 \mathrm{mmol} \mathrm{L}^{-1}$ linker for 30 min to ensure efficient binding of biomolecules as well as cancer markers onto the substrate. Biomolecules at various concentration levels were then injected into the microfluidic chamber and incubated for around $20 \mathrm{~min}$ at room temperature for lateral position shift signal collection.

\section{Results and Discussion}

\subsection{Evaluation of the Sensor Device}

A schematic illustration of our experimental setup is shown in Fig. 2a. The incident light beam from a He-Ne laser is split into $p$-polarized and $s$-polarized light beams through a polarized beam splitter. An optical chopper is used to ensure that only one of the polarizations can reach the sensing substrate in a fixed period time. A high refractive index prism was mounted on a translation stage with the beam fixed at the surface plasmon resonance dip angle for achieving maximum lateral position shift. The sensing substrate is integrated with a microfluidic chamber to realize convenient transportation of sample solutions using a syringe pump. The real-time positions of the $p$-polarized and $s$-polarized reflected light beams are recorded by a lateral position sensing detector. The lateral position signals are then collected through a data acquisition card and analyzed using a LABVIEW plus MATLAB program. During the biosensing processes, when sample liquids are pumped into the microfluidic chamber, we are able to conduct real-time measurement of the phase singularity-related lateral position shift, where the lateral shifts are induced by the binding of target molecules to the sensing surface.

To evaluate the performance of the 2D GST-on-Au sensing substrate, the angular scanning reflectivity spectra of $2 \mathrm{D}$ GST-on-Au and Au-only substrate in air were measured. The experimental results show good agreement with theoretical calculations, which confirms the reliability of our device and serves as a good calibration for the assessment of sensing performance. As shown in Fig. S4, the presence of GST material clearly leads to a deeper resonance dip (minimum intensity lowered by $50 \%$ ). The stronger zero-reflection effects also result in a much larger lateral position shift.

Further, as a standard sensor evaluation procedure, we sequentially injected glycerol solutions of different concentration levels into the microfluidic chamber while the reflectivity is monitored. Figure $2 b$ shows the signals acquired under different glycerol concentrations using atomically thin GST-on-Au substrate. The summarized lateral position shifts of glycerol solutions with concentration levels from 1 to 5\% for both Au-only substrate and 2D GST-on-Au substrate were also plotted. Experimental results confirm the significant enhancement in lateral position shift associated with the incorporation of atomically thin GST, which is in good agreement with our theoretical calculation. Also, the measured lateral position shift has a linear relationship with glycerol/water weight ratios. The measured signal change for $1 \%$ glycerol (0.0012 Refractive Index Unit, RIU) using atomically thin GST-on-Au substrate is $71.38 \mu \mathrm{m}$, which corresponds to a sensitivity figure-of-merit of $5.95 \times 10^{4} \mu \mathrm{m} /$ RIU. The position of $p$-polarized reflected light changes drastically while $s$-polarized reflected light remains in the same position upon injecting the glycerol/water solutions into the chamber as shown in Fig. S5. The signal is very stable due to the use of differential measurement scheme.

\subsection{Biomolecule Sensing}

It is known that the main challenge of optical detection lies in the detection of biomolecules with very low molecular weight (less than $400 \mathrm{Da}$ ) such as biotin with a molecular weight of $244 \mathrm{Da}$. Conventional plasmonic biosensors can only detect biotin molecules with concentration higher than 


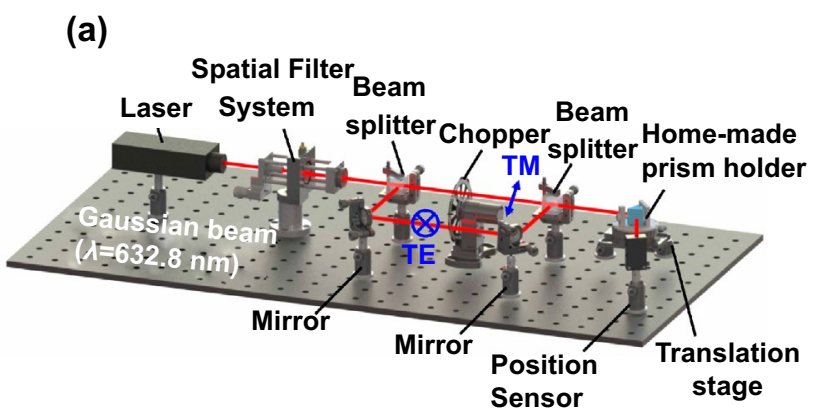

(b)
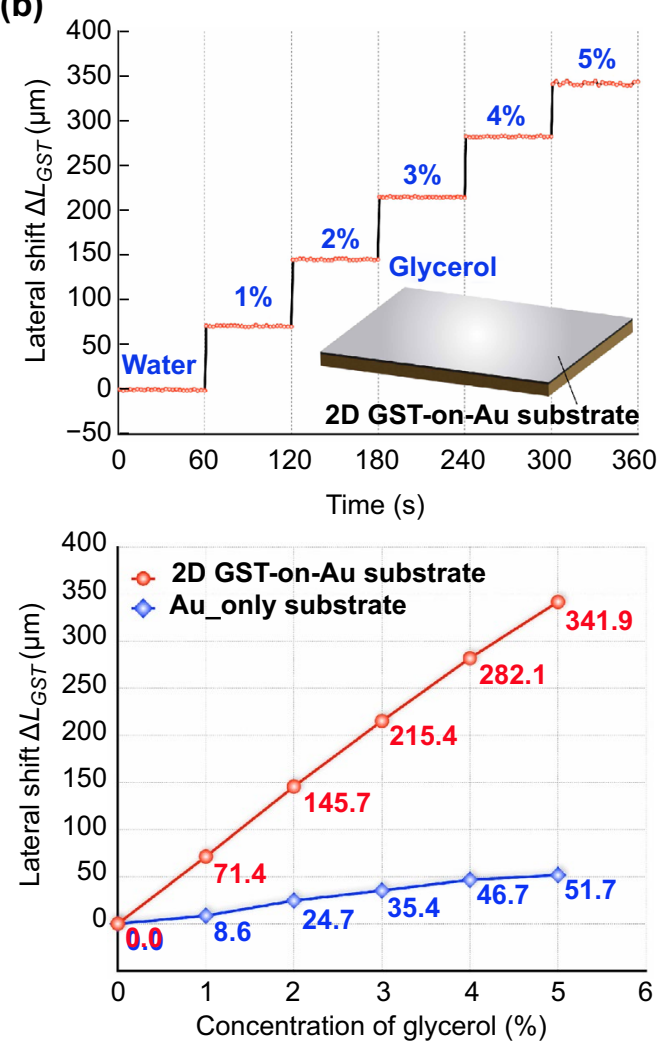
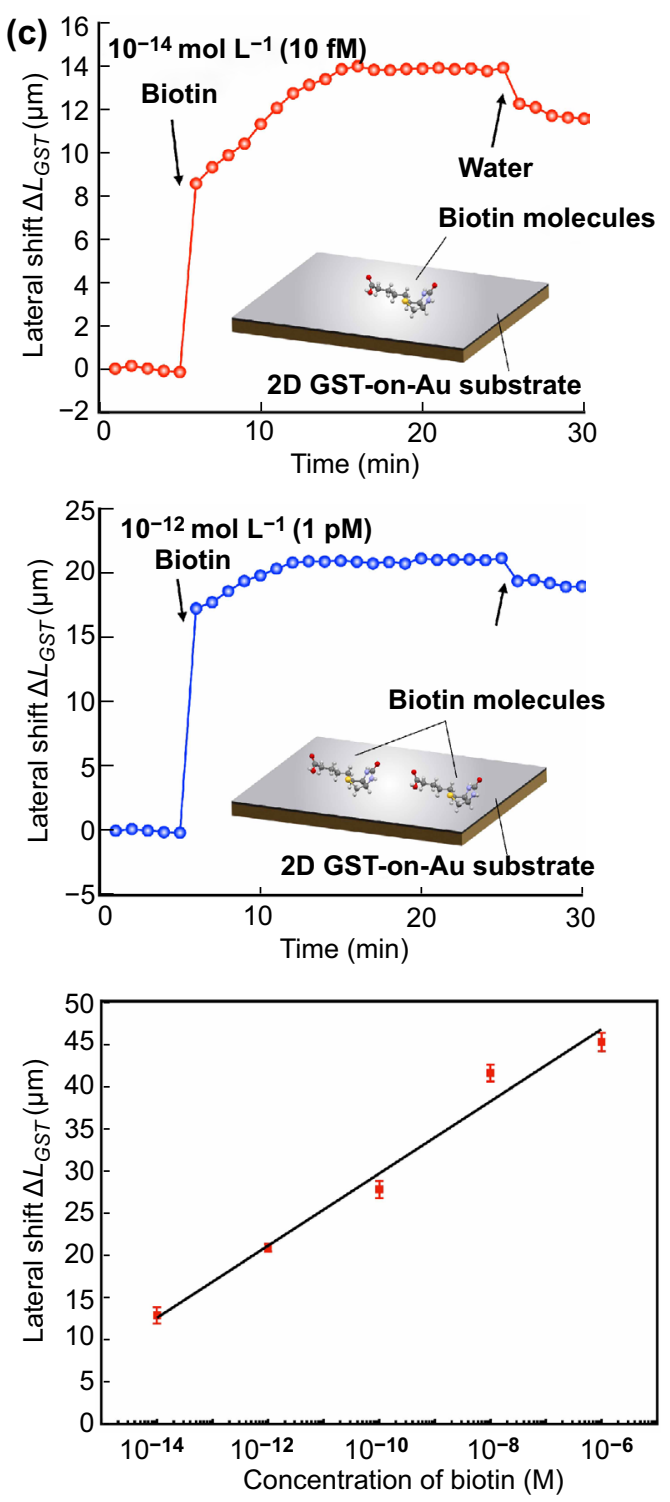

Fig. 2 a Optical setup for measuring differential lateral position shift between $p$ - and $s$-polarized light. b Evaluation results of measuring glycerol solutions. $\mathbf{c}$ Real-time detection of biotin molecules based on lateral position shift measurement

$100 \mu \mathrm{M}[61]$. With the recent development of nanomaterialenhanced plasmonic sensors, the detection limit has been lowered to nanomolar [62] or picomolar [63] level. In this work, we are able to further improve the detection limit by more than three orders of magnitude. Solutions of the biomolecules with different concentrations ranging from $10 \mathrm{fM}$ to $10 \mu \mathrm{M}$ were prepared. To ensure efficient binding to the 2D GST-on-Au substrate, we first injected a chemical linker (3-Aminopropyl) trimethoxysilane (APTMS) into the microfluidic chamber to functionalize the sensing substrate with alkoxysilane molecules. This was followed by sequential injection of biotin biomolecules at different molarities to the chamber, which then triggered the corresponding lateral position shifts. Figure $2 \mathrm{c}$ shows the detection of biotin molecules based on the measurement of lateral position shifts. The lateral position signal due to biotin binding changed with low speed after a step increase in concentration and saturated at around $14 \mu \mathrm{m}$ for a biotin concentration of 10 $\mathrm{fM}$. For biotin with a concentration of $1 \mathrm{pM}$, the lateral shift change shows a similar trend and stabilized at around $20 \mu \mathrm{m}$ after the saturation time (20 $\mathrm{min})$. This is quite impressive considering the low molecular weight and extremely low 
concentration level. After each detection run, we flushed away the excess biotin molecules not bound to the substrate. The lateral position shift barely changed, suggesting that most of the targeted molecules have been bound to the substrate. The observed change in lateral position shift is primarily attributed to the binding between the biomolecules and the substrate. The differential lateral position shift signals acquired under biotin concentrations ranging from $10^{-14}$ to $10^{-6} \mathrm{M}$ were also summarized, which shows that the lateral shift is linearly proportional to log scale of biotin concentration. Our experimental results have clearly shown that the proposed 2D GST-on-Au biosensor scheme exhibits an ultra-high sensitivity, which is of significance for realtime label-free biosensing.

Besides biotin molecules, we also detected bovine serum albumin (BSA) molecules, which have relatively high molecular weight $(66,463 \mathrm{Da})$, at different concentration levels based on the giant lateral position shift. In BSA sensing experiments, the lateral position shift shows an abrupt change immediately after the injection of BSA solutions. The minimum detectable concentration is estimated to be lower than $10 \mathrm{fM}$. The lateral position shift has increased to $48.34 \mu \mathrm{m}$ after a binding event with $10 \mathrm{fM}$ BSA. Solutions of the biomolecules with different concentrations ranging from $10 \mathrm{fM}$ to $10 \mu \mathrm{M}$ were detected and recorded in Fig. S6 based on lateral position shift measurement, which shows a linear increase in lateral position shifts with increasing BSA concentrations. Signal saturation will start when the concentration goes above $10^{-6} \mathrm{~mol} \mathrm{~L}^{-1}$. Given the large lateral position shift at $10 \mathrm{fM}$ levels, for both BSA and biotin, we can assert that this sensor has the capacity of sub-attomole detection sensitivity.

\subsection{Sub-attomole Cancer Biomarker Detection}

Further, to demonstrate the capability of this biosensor for clinical applications, we carried out TNF- $\alpha$ (tumor necrosis factor $\alpha$ ) antigen detection using a sandwich immunoassay strategy. In our experiment, we first immersed the sensing surface with capture antibodies. To block non-specific binding in the sensing surface, we flowed BSA molecules, which is widely used as blocking agent [64-66], into the microfluidic chamber in advance to block the unbound sites in the sensing substrate. Then an antigen-containing solution was injected into the microfluidic chamber. A rapidly increasing lateral position shift indicates the specific binding between antigen and antibody molecules. As shown in Fig. 3a, our experimental results confirm that the projected detection limit of our atomically thin GSTon-Au plasmonic biosensor can reach $10^{-15} \mathrm{M}$ (1 fM) for TNF- $\alpha$ antigen detection. The antigen at a quantity as low as 0.05 attomoles, corresponding to $1 \mathrm{fM}$ in a $50 \mu \mathrm{L}$ solution, can be measured. To better show the superior sensing capability of the proposed sensing device, a comparison experiment was conducted using Au substrate enhanced by $2 \mathrm{D} \mathrm{MoS}_{2}$ nanosheets (Fig. 3b). In this experiment, we flowed 2D $\mathrm{MoS}_{2}$ nanosheets, which is known as standard $2 \mathrm{D}$ materials that are used as the signal amplification nanotags [67-69], into the microfluidic chamber. With the use of $2 \mathrm{D} \mathrm{MoS}$ nanosheets, the lateral position shift signal can be increased to $16.04 \mu \mathrm{m}$ for $1 \mathrm{nM}$ cancer marker detection. Then with the injection of antibody-containing solution, which binds the antigen at a different epitope than the capture antibody, the lateral position shift will increase again, thus further enhancing the sensing signal. However, even under careful optimization of the $2 \mathrm{D} \mathrm{MoS}_{2}$ nanosheets-onAu substrate, which is much more costly than that case of atomically thin GST, the sensitivity enhancement is still only approximately one-third of our proposed GST-coated substrate. As shown in Fig. 3c, the lateral shift enhanced by GST-on-Au substrate can reach more than $50 \mu \mathrm{m}$ after the binding of $1 \mathrm{nM}$ cancer marker biosamples. Under the same configuration, we also conducted experiments on Au-only sensing substrate. Experimental results showed that there is hardly any identifiable lateral position shift change upon injection of the biosamples (insert in Fig. 3c). In addition to the remarkable sensitivity, the specificity of our sensing device has also been verified through comparing this specific binding process with the non-specific binding between TNF-alpha and BSA molecules. The lateral position shift signal when flowing BSA with a large concentration ( $10^{5}$ times higher than anti-TNF antibody) only increased to $4.37 \mu \mathrm{m}$, which is much smaller compared to the signal acquired after flowing monoclonal anti-TNF antibody $(16.70 \mu \mathrm{m})$, as shown in Fig. S7. The experimental results indicate that our sensing device also has great potential in realizing high-specificity detection in addition to the ultra-high sensitivity. 

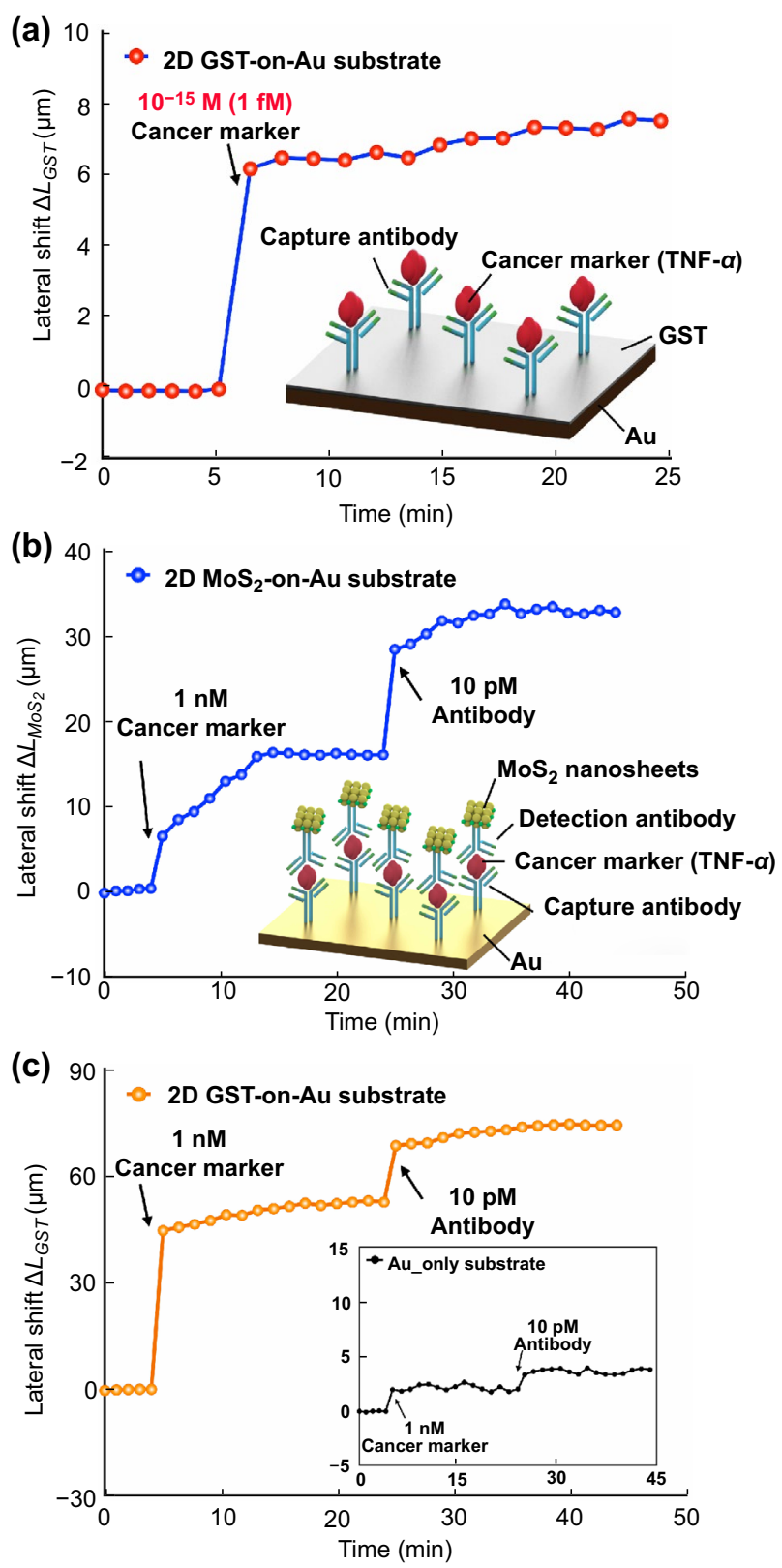

Fig. 3 a Detection of extremely low (1 fM) cancer marker concentration using 2D GST-on-Au substrate. b Detection of 1nм cancer marker using 2D $\mathrm{MoS}_{2}$-on-Au substrate. c Detection of 1nм cancer marker using 2D GST-on-Au substrate with insert figure showing the performance of Au-only substrate

\section{Conclusions}

In this paper, we present a new plasmonic biosensor with significantly enhanced sensitivity due to the giant lateral position associated with a phase singularity. The sensing substrate is constructed by depositing an atomically thin
$\mathrm{Ge}_{2} \mathrm{Sb}_{2} \mathrm{Te}_{5}$ phase change nanomaterial on gold thin film. The high absorbance of GST material significantly suppresses the reflectivity to a point close to the topological darkness, which yields to extremely steep phase change and further results in a giant lateral position shift with respect to refractive index variations. Refractive index sensing experiments involving the use of glycerol solutions at different concentration levels shows a sensitivity figure-of-merit of $5.95 \times 10^{4} \mu \mathrm{m} / \mathrm{RIU}$. The maximum detectable lateral position shift is $341.90 \mu \mathrm{m}$, which is the highest ever reported in the literature to our best knowledge. Furthermore, we have demonstrated the capability of our device for biosensing using biotin (small molecules) and BSA (large molecules). The experimental detection limit for light molecules (biotin, $244.31 \mathrm{Da}$ ) is in the order of $10^{-14} \mathrm{M}$. This is a significant improvement given that most existing SPR systems are only achieving $\mathrm{pM}$ detection sensitivity limit for low molecular weight biomolecules. Our experiments also reveal that atomically GST layer exhibits three times better improvement than $2 \mathrm{D} \mathrm{MoS}_{2}$ nanosheets, which is commonly used as the signal amplification nanotags. The proposed plasmonic device also shows excellent performance for cancer marker detection. The detection limit has been experimentally demonstrated to be $10^{-15} \mathrm{M}$ for TNF- $\alpha$ cancer marker, which is orders of magnitude higher than most label-free detection methods. To summarize, the reported integration of atomically GST layer with plasmonic substrate has been shown to be useful for ultrasensitive biosensing applications with subattomole detection limit. It is our view that this label-free, real-time, highly sensitive biosensor has great potential for monitoring chemical and biological reactions with ultra-high sensitivity especially for the clinical diagnostic applications.

Acknowledgements We thank Shiyue Liu from School of Life Sciences in The Chinese University of Hong Kong for helpful discussions. This work is supported under the PROCORE-France/ Hong Kong Joint Research Scheme (F-CUHK402/19) and by the Research Grants Council, Hong Kong Special Administration Region (AoE/P-02/12, 14210517, 14207419, N_CUHK407/16). This project has received funding from the European Union's Horizon 2020 research and innovation programme under the Marie Sklodowska-Curie Grant Agreement No. 798916. Y. Wang is supported under the Hong Kong PhD Fellowship Scheme.

Open Access This article is licensed under a Creative Commons Attribution 4.0 International License, which permits use, sharing, adaptation, distribution and reproduction in any medium or format, as long as you give appropriate credit to the original author(s) and the source, provide a link to the Creative Commons licence, and 
indicate if changes were made. The images or other third party material in this article are included in the article's Creative Commons licence, unless indicated otherwise in a credit line to the material. If material is not included in the article's Creative Commons licence and your intended use is not permitted by statutory regulation or exceeds the permitted use, you will need to obtain permission directly from the copyright holder. To view a copy of this licence, visit http://creativecommons.org/licenses/by/4.0/.

Supplementary Information The online version contains supplementary material available at https://doi.org/10.1007/s40820-02100613-7.

\section{References}

1. N. Cheng, D. Du, X. Wang, D. Liu, W. Xu et al., Recent advances in biosensors for detecting cancer-derived exosomes. Trends Biotechnol. 37, 1236-1254 (2019). https://doi.org/10. 1016/j.tibtech.2019.04.008

2. Z. Wang, W. Cui, Crispr-cas system for biomedical diagnostic platforms. View 1, 200008 (2020). https://doi.org/10.1002/ viw. 20200008

3. G. Yang, Z. Xiao, C. Tang, Y. Deng, H. Huang et al., Recent advances in biosensor for detection of lung cancer biomarkers. Biosens. Bioelectron. 141, 111416 (2019). https://doi.org/10. 1016/j.bios.2019.111416

4. M. Yamashita, E. Passegue, TNF-alpha coordinates hematopoietic stem cell survival and myeloid regeneration. Cell Stem Cell 25, 357-372 (2019). https://doi.org/10.1016/j.stem. 2019.05.019

5. J. Silke, E.L. Hartland, Masters, marionettes and modulators: Intersection of pathogen virulence factors and mammalian death receptor signaling. Curr. Opin. Immunol. 25, 436-440 (2013). https://doi.org/10.1016/j.coi.2013.05.011

6. D. Brenner, H. Blaser, T.W. Mak, Regulation of tumour necrosis factor signalling: Live or let die. Nat. Rev. Immunol. 15, 362-374 (2015). https://doi.org/10.1038/nri3834

7. B. Ungar, I. Levy, Y. Yavne, M. Yavzori, O. Picard et al., Optimizing anti-TNF- $\alpha$ therapy: Serum levels of infliximab and adalimumab are associated with mucosal healing in patients with inflammatory bowel diseases. Clin. Gastroenterol. Hepatol. 14, 550-557.e552 (2016). https://doi.org/10.1016/j.cgh. 2015.10.025

8. Y. Wang, J. Xu, X. Zhang, C. Wang, Y. Huang et al., TNF- $\alpha-$ induced LRG1 promotes angiogenesis and mesenchymal stem cell migration in the subchondral bone during osteoarthritis. Cell Death Dis. 8, e2715 (2017). https://doi.org/10.1038/cddis. 2017.129

9. M. Kapoor, J. Martel-Pelletier, D. Lajeunesse, J.P. Pelletier, H. Fahmi, Role of proinflammatory cytokines in the pathophysiology of osteoarthritis. Nat. Rev. Rheumatol. 7, 33-42 (2011). https://doi.org/10.1038/nrrheum.2010.196

10. Q. Zhang, D. Dehaini, Y. Zhang, J. Zhou, X. Chen et al., Neutrophil membrane-coated nanoparticles inhibit synovial inflammation and alleviate joint damage in inflammatory arthritis. Nat. Nanotechnol. 13, 1182-1190 (2018). https:// doi.org/10.1038/s41565-018-0254-4

11. H.A. Sahibzada, Z. Khurshid, R.S. Khan, M. Naseem, K.M. Siddique et al., Salivary IL-8, IL-6 and TNF-alpha as potential diagnostic biomarkers for oral cancer. Diagnostics 7(2), 21 (2017). https://doi.org/10.3390/diagnostics7020021

12. Y. Ma, Y. Ren, Z.J. Dai, C.J. Wu, Y.H. Ji et al., IL-6, IL-8 and TNF-alpha levels correlate with disease stage in breast cancer patients. Adv. Clin. Exp. Med. 26, 421-426 (2017). https://doi. org/10.17219/acem/62120

13. C. Agnoli, S. Grioni, V. Pala, A. Allione, G. Matullo et al., Biomarkers of inflammation and breast cancer risk: A casecontrol study nested in the epic-varese cohort. Sci. Rep. 7, 12708 (2017). https://doi.org/10.1038/s41598-017-12703-x

14. E. Dalaveris, T. Kerenidi, A. Katsabeki-Katsafli, T. Kiropoulos, K. Tanou et al., VEGF, TNF-alpha and 8-isoprostane levels in exhaled breath condensate and serum of patients with lung cancer. Lung Cancer 64, 219-225 (2009). https://doi.org/ 10.1016/j.lungcan.2008.08.015

15. M. Komatsu, D. Kobayashi, K. Saito, D. Furuya, A. Yagihashi et al., Tumor necrosis factor-a in serum of patients with inflammatory bowel disease as measured by a highly sensitive immuno-PCR. Clin. Chem. 47, 1297-1301 (2001). https://doi. org/10.1093/clinchem/47.7.1297

16. G. La Rocca, S.H. Olejniczak, A.J. Gonzalez, D. Briskin, J.A. Vidigal et al., In vivo, argonaute-bound micrornas exist predominantly in a reservoir of low molecular weight complexes not associated with mRNA. Proc. Natl. Acad. Sci. USA 112, 767-772 (2015). https://doi.org/10.1073/pnas.1424217112

17. W. Hu, G. He, H. Zhang, X. Wu, J. Li et al., Polydopaminefunctionalization of graphene oxide to enable dual signal amplification for sensitive surface plasmon resonance imaging detection of biomarker. Anal. Chem. 86, 4488-4493 (2014). https://doi.org/10.1021/ac5003905

18. S.B. Nimse, M.D. Sonawane, K.S. Song, T. Kim, Biomarker detection technologies and future directions. Analyst 141, 740-755 (2016). https://doi.org/10.1039/c5an01790d

19. V. Jayanthi, A.B. Das, U. Saxena, Recent advances in biosensor development for the detection of cancer biomarkers. Biosens. Bioelectron. 91, 15-23 (2017). https://doi.org/10.1016/j. bios.2016.12.014

20. S. Yadav, N. Kashaninejad, M.K. Masud, Y. Yamauchi, N.T. Nguyen et al., Autoantibodies as diagnostic and prognostic cancer biomarker: Detection techniques and approaches. Biosens. Bioelectron. 139, 111315 (2019). https://doi.org/10. 1016/j.bios.2019.111315

21. H. Filik, A.A. Avan, Nanostructures for nonlabeled and labeled electrochemical immunosensors: Simultaneous electrochemical detection of cancer markers: A review. Talanta 205, 120153 (2019). https://doi.org/10.1016/j.talanta.2019. 120153

22. L. Miccio, F. Cimmino, I. Kurelac, M.M. Villone, V. Bianco et al., Perspectives on liquid biopsy for label-free detection of "circulating tumor cells" through intelligent lab-on-chips. 
View 1, 20200034 (2020). https://doi.org/10.1002/viw.20200 034

23. L. Huang, D.D. Gurav, S. Wu, W. Xu, V. Vedarethinam et al., A multifunctional platinum nanoreactor for point-of-care metabolic analysis. Matter 1, 1669-1680 (2019). https://doi. org/10.1016/j.matt.2019.08.014

24. C. Pei, C. Liu, Y. Wang, D. Cheng, R. Li et al., FeOOH@ metal-organic framework core-satellite nanocomposites for the serum metabolic fingerprinting of gynecological cancers. Angew. Chem. Int. Ed. 59, 10831-10835 (2020). https://doi. org/10.1002/anie.202001135

25. W. Shu, Y. Wang, C. Liu, R. Li, C. Pei et al., Construction of a plasmonic chip for metabolic analysis in cervical cancer screening and evaluation. Small Methods 4, 1900469 (2020). https://doi.org/10.1002/smtd.201900469

26. J. Cao, X. Shi, D.D. Gurav, L. Huang, H. Su et al., Metabolic fingerprinting on synthetic alloys for medulloblastoma diagnosis and radiotherapy evaluation. Adv. Mater. 32, e2000906 (2020). https://doi.org/10.1002/adma.202000906

27. L. Huang, L. Wang, X. Hu, S. Chen, Y. Tao et al., Machine learning of serum metabolic patterns encodes early-stage lung adenocarcinoma. Nat. Commun. 11, 3556 (2020). https://doi. org/10.1038/s41467-020-17347-6

28. H. Sipova, J. Homola, Surface plasmon resonance sensing of nucleic acids: A review. Anal. Chim. Acta 773, 9-23 (2013). https://doi.org/10.1016/j.aca.2012.12.040

29. P. Singh, Spr biosensors: Historical perspectives and current challenges. Sens. Actuat. B: Chem. 229, 110-130 (2016). https://doi.org/10.1016/j.snb.2016.01.118

30. D.E.P. Souto, J. Volpe, C.C. Goncalves, C.H.I. Ramos, L.T. Kubota, A brief review on the strategy of developing SPRbased biosensors for application to the diagnosis of neglected tropical diseases. Talanta 205, 120122 (2019). https://doi.org/ 10.1016/j.talanta.2019.120122

31. S. Zeng, D. Baillargeat, H.P. Ho, K.T. Yong, Nanomaterials enhanced surface plasmon resonance for biological and chemical sensing applications. Chem. Soc. Rev. 43, 3426-3452 (2014). https://doi.org/10.1039/c3cs60479a

32. B.P. Nelson, A.G. Frutos, J.M. Brockman, R.M. Corn, Nearinfrared surface plasmon resonance measurements of ultrathin films. 1. Angle shift and SPR imaging experiments. Anal. Chem. 71, 3928-3934 (1999). https://doi.org/10.1021/ac990 $517 \mathrm{x}$

33. S. Wang, X. Shan, U. Patel, X. Huang, J. Lu et al., Label-free imaging, detection, and mass measurement of single viruses by surface plasmon resonance. Proc. Natl. Acad Sci. USA 107, 16028-16032 (2010). https://doi.org/10.1073/pnas.10052 64107

34. J. Cao, M.H. Tu, T. Sun, K.T.V. Grattan, Wavelength-based localized surface plasmon resonance optical fiber biosensor. Sens. Actuat. B: Chem. 181, 611-619 (2013). https://doi.org/ 10.1016/j.snb.2013.02.052

35. E. Kazuma, T. Tatsuma, Localized surface plasmon resonance sensors based on wavelength-tunable spectral dips. Nanoscale 6, 2397-2405 (2014). https://doi.org/10.1039/c3nr05846h
36. G. Xu, M. Tazawa, P. Jin, S. Nakao, K. Yoshimura, Wavelength tuning of surface plasmon resonance using dielectric layers on silver island films. Appl. Phys. Lett. 82, 3811-3813 (2003). https://doi.org/10.1063/1.1578518

37. V.G. Kravets, F. Schedin, R. Jalil, L. Britnell, R.V. Gorbachev et al., Singular phase nano-optics in plasmonic metamaterials for label-free single-molecule detection. Nat. Mater. 12, 304-309 (2013). https://doi.org/10.1038/nmat3537

38. Y.H. Huang, H.P. Ho, S.Y. Wu, S.K. Kong, Detecting phase shifts in surface plasmon resonance: A review. Adv. Optical Technol. 2012, 1-12 (2012). https://doi.org/10.1155/2012/ 471957

39. F. Yesilkoy, R.A. Terborg, J. Pello, A.A. Belushkin, Y. Jahani et al., Phase-sensitive plasmonic biosensor using a portable and large field-of-view interferometric microarray imager. Light Sci. Appl. 7, 17152-17152 (2017). https://doi.org/10. 1038/1sa.2017.152

40. C. Coutant, S. Ravaine, X. Wang, J. Toudert, V. Ponsinet et al., Plasmonic metamaterials for ultra-sensitive sensing: Topological darkness. Rendiconti Lincei 26, 175-182 (2015). https:// doi.org/10.1007/s12210-015-0404-7

41. L. Malassis, P. Masse, M. Treguer-Delapierre, S. Mornet et al., Topological darkness in self-assembled plasmonic metamaterials. Adv. Mater. 26, 324-330 (2014). https://doi.org/10.1002/ adma.201303426

42. Q. You, Y. Shan, S. Gan, Y. Zhao, X. Dai et al., Giant and controllable goos-hänchen shifts based on surface plasmon resonance with graphene- $\mathrm{MoS}_{2}$ heterostructure. Opt. Mater. Express 8, 3036-3048 (2018). https://doi.org/10.1364/ome.8. 003036

43. X. Yin, L. Hesselink, Goos-hänchen shift surface plasmon resonance sensor. Appl. Phys. Lett. 89, 261108 (2006). https:// doi.org/10.1063/1.2424277

44. T. Cao, C. Wei, R.E. Simpson, L. Zhang, M.J. Cryan, Rapid phase transition of a phase-change metamaterial perfect absorber. Opt. Mater. Express 3, 1101-1110 (2013). https:// doi.org/10.1364/ome.3.001101

45. D.G. Baranov, A. Krasnok, T. Shegai, A. Alù, Y. Chong, Coherent perfect absorbers: Linear control of light with light. Nat. Rev. Mater. 2, 17064 (2017). https://doi.org/10.1038/natre vmats.2017.64

46. C. Xu, J. Xu, G. Song, C. Zhu, Y. Yang et al., Enhanced displacements in reflected beams at hyperbolic metamaterials. Opt. Express 24, 21767-21776 (2016). https://doi.org/10. 1364/OE.24.021767

47. R.-G. Wan, M.S. Zubairy, Tunable and enhanced GoosHänchen shift via surface plasmon resonance assisted by a coherent medium. Opt. Express 28, 6036-6047 (2020). https:// doi.org/10.1364/oe.384419

48. J.H. Kang, S. Wang, Z. Shi, W. Zhao, E. Yablonovitch et al., Goos-Hanchen shift and even-odd peak oscillations in edgereflections of surface polaritons in atomically thin crystals. Nano Lett. 17, 1768-1774 (2017). https://doi.org/10.1021/acs. nanolett.6b05077 
49. Y. Xu, C.T. Chan, H. Chen, Goos-Hanchen effect in epsilonnear-zero metamaterials. Sci. Rep. 5, 8681 (2015). https://doi. org/10.1038/srep08681

50. X. Ding, W. Cheng, Y. Li, J. Wu, X. Li et al., An enzyme-free surface plasmon resonance biosensing strategy for detection of DNA and small molecule based on nonlinear hybridization chain reaction. Biosens. Bioelectron. 87, 345-351 (2017). https://doi.org/10.1016/j.bios.2016.08.077

51. G. Qiu, S.P. Ng, C.L. Wu, Label-free surface plasmon resonance biosensing with titanium nitride thin film. Biosens. Bioelectron. 106, 129-135 (2018). https://doi.org/10.1016/j.bios. 2018.02.006

52. A. Kumar, G. Sachdeva, R. Pandey, S.P. Karna, Optical absorbance in multilayer two-dimensional materials: Graphene and antimonene. Appl. Phys. Lett. 116, 263102 (2020). https:// doi.org/10.1063/5.0010794

53. K.S. Novoselov, A. Mishchenko, A. Carvalho, A.H. Castro Neto, 2D materials and van der waals heterostructures. Science 353, aac9439 (2016). https://doi.org/10.1126/science. aac9439

54. Z. Sun, A. Martinez, F. Wang, Optical modulators with 2D layered materials. Nat. Photonics 10, 227-238 (2016). https:// doi.org/10.1038/nphoton.2016.15

55. C.-F. Li, Negative lateral shift of a light beam transmitted through a dielectric slab and interaction of boundary effects. Phys. Rev. Lett. 91, 133903 (2003). https://doi.org/10.1103/ PhysRevLett.91.133903

56. F. Yesilkoy, R.A. Terborg, J. Pello, A.A. Belushkin, Y. Jahani et al., Phase-sensitive plasmonic biosensor using a portable and large field-of-view interferometric microarray imager. Light Sci. Appl. 7, 17152-17152 (2018). https://doi.org/10. 1038/lsa.2017.152

57. F. Feng, G. Si, C. Min, X. Yuan, M. Somekh, On-chip plasmonic spin-hall nanograting for simultaneously detecting phase and polarization singularities. Light Sci. Appl. 9, 95 (2020). https://doi.org/10.1038/s41377-020-0330-z

58. W. Wu, W. Zhang, S. Chen, X. Ling, W. Shu et al., Transitional Goos-Hanchen effect due to the topological phase transitions. Opt. Express 26, 23705-23713 (2018). https://doi.org/10. 1364/OE.26.023705

59. C. Luo, J. Guo, Q. Wang, Y. Xiang, S. Wen, Electrically controlled Goos-Hanchen shift of a light beam reflected from the metal-insulator-semiconductor structure. Opt. Express 21, 10430-10439 (2013). https://doi.org/10.1364/OE.21.010430
60. S. Lee, L. Li, Rapid super-resolution imaging of sub-surface nanostructures beyond diffraction limit by high refractive index microsphere optical nanoscopy. Opt. Commun. 334, 253-257 (2015). https://doi.org/10.1016/j.optcom.2014.08. 048

61. A.V. Kabashin, P. Evans, S. Pastkovsky, W. Hendren, G.A. Wurtz et al., Plasmonic nanorod metamaterials for biosensing. Nat. Mater. 8, 867-871 (2009). https://doi.org/10.1038/nmat2 546

62. M. Focsan, A. Campu, A.-M. Craciun, M. Potara, C. Leordean et al., A simple and efficient design to improve the detection of biotin-streptavidin interaction with plasmonic nanobiosensors. Biosens. Bioelectron. 86, 728-735 (2016). https://doi.org/10. 1016/j.bios.2016.07.054

63. K.V. Sreekanth, Y. Alapan, M. ElKabbash, E. Ilker, M. Hinczewski et al., Extreme sensitivity biosensing platform based on hyperbolic metamaterials. Nat. Mater. 15, 621-627 (2016). https://doi.org/10.1038/nmat4609

64. R. Ahirwar, S. Bariar, A. Balakrishnan, P. Nahar, Bsa blocking in enzyme-linked immunosorbent assays is a non-mandatory step: A perspective study on mechanism of bsa blocking in common elisa protocols. RSC Adv. 5, 100077-100083 (2015). https://doi.org/10.1039/c5ra20750a

65. J. Lee, I.S. Park, H. Kim, J.S. Woo, B.S. Choi et al., BSA as additive: A simple strategy for practical applications of PNA in bioanalysis. Biosens. Bioelectron. 69, 167-173 (2015). https://doi.org/10.1016/j.bios.2015.02.030

66. R. Wang, X. Zhou, X. Zhu, C. Yang, L. Liu et al., Isoelectric bovine serum albumin: Robust blocking agent for enhanced performance in optical-fiber based DNA sensing. ACS Sens. 2, 257-262 (2017). https://doi.org/10.1021/acssensors.6b007 46

67. S. Manzeli, D. Ovchinnikov, D. Pasquier, O.V. Yazyev, A. Kis, 2D transition metal dichalcogenides. Nat. Rev. Mater. 2, 17033 (2017). https://doi.org/10.1038/natrevmats.2017.33

68. C. Tan, P. Yu, Y. Hu, J. Chen, Y. Huang et al., High-yield exfoliation of ultrathin two-dimensional ternary chalcogenide nanosheets for highly sensitive and selective fluorescence DNA sensors. J. Am. Chem. Soc. 137, 10430-10436 (2015). https://doi.org/10.1021/jacs.5b06982

69. S. Zeng, S. Hu, J. Xia, T. Anderson, X.-Q. Dinh et al., Graphene- $\mathrm{MoS}_{2}$ hybrid nanostructures enhanced surface plasmon resonance biosensors. Sens. Actuat. B: Chem. 207, 801-810 (2015). https://doi.org/10.1016/j.snb.2014.10.124 